\title{
Preface: Scope of Logic Theorems In Memoriam Adolf Lindenbaum
}

\author{
Jean-Yves Beziau
}

In view of the speedy and huge expansion of the universe of logics, the question of the scope of validity and the domain of application of fundamental logic theorems is more than ever crucial. What is true for classical logic and theories based on it, does not necessarily hold for non-classical logics.

But we may wonder if there is a logic deserving the name in which a theorem such as the incompleteness theorem does not hold. On the other hand a theorem such as cut-elimination does not hold for many interesting logical systems. Cut-elimination expresses the intrinsic analycity of a logic, the fact that a proof of a theorem depends only of its constituents, a not always welcome feature. Anyway, it is interesting to find necessary and/or sufficient conditions for cut-elimination to hold. And also for any important theorem of logic.

What are the most important theorems of modern logic? We can establish the following list:

1. Löwenheim-Skolem (1915-1920)

2. Completeness (Post 1921-Gödel 1930)

3. Incompleteness one and two (Gödel 1931)

4. Cut-elimination (Gentzen 1934)

5. Undefinability (Tarski 1936)

6. Undecidability (Church-Turing 1936)

7. Lindenbaum maximalization theorem (1937)

8. Compactness (Malcev 1938)

9. Incompleteness of finite matrices for some modal logics (Dugundji 1940)

10. Beth's definability theorem (1953)

11. Craig's interpolation theorem (1957)

12. Completeness of relational semantics for some modal logics (Kripke 1959)

13. Independence of the continuum hypothesis (Cohen 1963)

14. Lindström's theorem (1969)

15. Paris-Harrington theorem (1977) 
The papers in this issue deal with some of these theorems. We intend to produce later on another special issue completing this first one.

Scope of logic theorems was the topic of the contest of the 4th World Congress on Universal Logic that took place in Rio de Janeiro, Brazil, April 3-7, 2013. The members of the jury were Yuri Gurevich, Daniele Mundici and Hiroakira Ono. The winner was Nathanael Leedom Ackerman. The paper he presented at UNILOG'2013 is included here.

This special issue is dedicated to Adolf Lindenbaum (1904-1941), a friend and colleague of Alfred Teitelbaum, aka Alfred Tarski. His work is still not very well-known. Lindenbaum has proved many theorems, but also has developed many new ideas and concepts. We are publishing here a paper by Jan Zygmunt about the life and work of Lindenbaum including a detailed bibliography of his work.

Lindenbaum wrote the following:

A mathematician, a modern mathematician in particular, is, as it would be said, in a superior degree of conscious activity: he is not only interested in the question of the what, but also in that of the how. He almost never restricts himself to a solution tout court of a problem. He always wants to have the most ??? solutions. Most what? The easiest, the shortest, the most general, etc. [2]

There are different proofs of the same theorem. It is interesting to present proofs which clearly distinguish what is general from what is particular. Making this distinction leads to some general theorems that can then be adapted to specific situations. This strategy has been developed in modern mathematics and it can be further developed in logic, this is the spirit of universal logic.

For example, Lindenbaum maximalization theorem saying that a noncontradictory theory can be extended in a maximal non-contradictory theory can be presented without referring to any specific logical language. This is done in particular by replacing the notion of non-contradiction based on negation, by the notion of non-trivial theory, a theory from which at least one formula is not deducible. This theorem can then be applied to many different logical systems, some not having negations, some having paraconsistent negations, etc. (see [1]).

\section{References}

[1] Beziau, J.-Y.: La véritable portée du théorème de Lindenbaum-Asser. Log. et Anal. 167-168, 341-359 (1999)

[2] Lindenbaum, A.: Sur la simplicité formelle des notions. In: Actes du congrès international de philosophie scientifique, vol. VII, pp. 28-38. Logique, Hermann (1936)

Jean-Yves Beziau

UFRJ-University of Brazil

CNPq-Brazilian Research Council

Rio de Janeiro, Brazil

e-mail: jean-yves.beziau@logica-universalis.org 